\title{
A Structural Pattern Analysis Approach to Iris Recognition
}

\author{
Hugo Proença \\ Department of Computer Science, IT - Networks and Multimedia Group \\ University of Beira Interior, Covilhã, Portugal \\ hugomcpedi.ubi.pt
}

Summary. Continuous efforts have been made in searching for robust and effective iris coding methods, since Daugman's pioneering work on iris recognition was published. Proposed algorithms follow the statistical pattern recognition paradigm and encode the iris texture information through phase, zero-crossing or texture-analysis based methods. In this paper we propose an iris recognition algorithm that follows the structural (syntactic) pattern recognition paradigm, which can be advantageous essentially for the purposes of description and of the human-perception of the system's functioning. Our experiments, that were performed on two widely used iris image databases (CASIA.v3 and ICE), show that the proposed iris structure provides enough discriminating information to enable accurate biometric recognition, while maintains the advantages intrinsic to structural pattern recognition systems.

Keywords: iris recognition, structural pattern analysis, biometrics.

\section{Introduction}

In 1987, Flom and Safir observed the stability of iris morphology over human lifetime and estimated the probability for the existence of two similar irises at 1 in $10^{72}$. Since then, the use of iris-based biometrics has been increasingly encouraged by both government and private entities. The iris is accepted as one of the most reliable biometric traits: it has a random morphogenesis and, apparently, no genetic penetrance.

The published iris recognition algorithms, at least the most relevant, follow the statistical Pattern Recognition (PR) paradigm (e.g., Daugman [3], Wildes [9] and Ma et al. [7]). They represent patterns as sets of features and regard them as points in a $d$-dimensional space. In this context, effectiveness is determined by the classes separability that is given by some data projection.

The structural PR approach is based upon the view that each pattern is composed of simple sub-patterns. The most elementary sub-patterns are the primitives. A pattern can be expressed in term of primitives and of relationships between primitives. Consequently, symbolic data structures (e.g., strings, tress, and graphs) are used for pattern representation. Feature sets generated by structural PR approaches contain 
a varying number of features. As the relationships between the primitives are also encoded, the feature vector must include additional components describing them or take an alternate form, such as a relational graph. Further, a pattern can be labeled as belonging to a class if its graph representation is isomorphic with some prototype graphs of the class [4].

This is intuitively appealing to biometrics because, apart classification, it simultaneously provides the description of how a given pattern is constructed from the primitives and the justification for a match/non-match produced in the comparison between two patterns. However, it is usually considered that may yields an enormous number of possibilities to be evaluated, demanding large training sets and very large computational efforts.

Our main goal is to show that structural-based iris recognition methods achieve very low error rates and be used for biometric purposes. Due to their intrinsic characteristics, this kind of methods facilitate the human-perception of the pattern encoding and matching, which can be useful - for instance - for forensic or security purposes. Also, the fact that our proposal does not contain any user-parameterized thresholds must be enhanced, regarding the dynamics of the imaging environments and the heterogeneity of the images that are presented to recognition systems.

The remainder of this paper is organized as follows: section 2 briefly summarizes the most cited iris recognition methods. A detailed description of the proposed method is given in section 3. Section 4 reports the experiments and results and, finally, section 5 concludes this paper.

\section{Iris Recognition}

As previously stated, the most relevant iris recognition algorithms follow the statistical PR paradigm and share the structure given in figure 1a. After the segmentation and normalization of the iris images, the iris data is encoded through one of three major variants: phase-based methods (e.g., [3]), zero-crossing methods (e.g., [2]), and texture-analysis methods (e.g., [9]). Daugman [3] used multi-scale quadrature wavelets to extract texture phase information and obtain an iris signature with 2048 binary components. Boles and Boashash [2] computed the zero-crossing representation of a 1D wavelet at different resolutions of concentric circles. Wildes [9] proposed the characterization of the iris texture through a Laplacian pyramid with four different levels (scales). Further, the comparison between iris signatures is performed and produced a numeric dissimilarity value that determines the subjects' identity. It is common to apply different distance metrics (Hamming [3], Euclidean [5] or weighted Euclidean [7]), or methods based on signal correlation [9].

Figure $1 \mathrm{~b}$ gives the block diagram of the proposed iris recognition method. According the structural PR paradigm, it starts by the detection of the primitive pixels among the iris data, followed by the detection of relationships between these primitives. This gives us a directed graph that represents the structure of the iris image and, as our experiments confirm, contains enough discriminating information to accurately distinguish between individuals. 


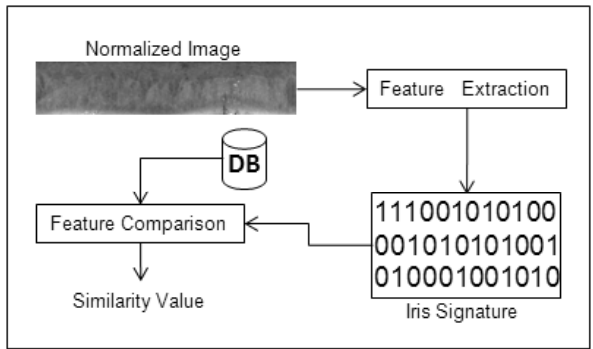

(a) Typical stages of statistical iris recognition.

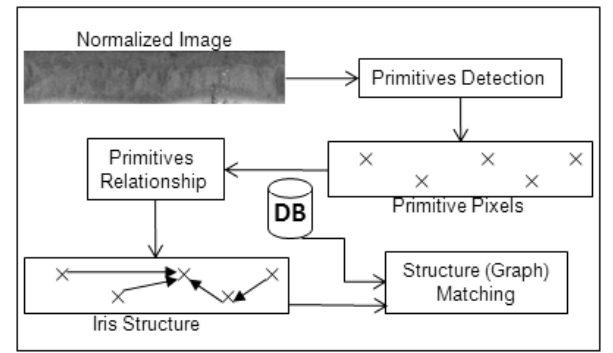

(b) Stages of the proposed recognition method, according to the structural pattern recognition paradigm.

Fig. 1. Statistical versus structural approaches to iris recognition.

\section{Underlying Iris Structure and Structural Matching}

Due to the pupils' varying sizes and to different distances, angles and optical magnifications of the imaging frameworks, the size of the irises in the captured images will have significant variations. As described by Daugman [3], the invariance to all these factors can be obtained through the translation of the captured data into a double dimensionless polar coordinate system, in a process known as the "Daugman Rubber Sheet". To each point on the iris, regardless of its size and pupillary dilation, it is assigned a pair of real coordinates $(r, \theta)$, where $r$ is on the unit interval $[0,1]$ and $\theta$ is an angle in $[0,2 \pi]$. The remapping of the iris image from raw cartesian coordinates $(x, y)$ to the dimensionless non concentric polar coordinate system $(r, \theta)$ is given by $I(x(r, \theta), y(r, \theta)) \rightarrow I(r, \theta)$, where $x(r, \theta)$ and $y(r, \theta)$ are linear combinations of both the set of pupillary and limbus boundary points, detected in the iris segmentation stage.

\subsection{Primitives}

The rationale behind our proposal is to consider as primitives the centers of the most homogeneous iris regions. These have lower average differences of intensities between neighbor pixels and are intuitively visually relevant regions. Let $I$ be a segmented and normalized iris image. Let $G(x, y)$ be a Gaussian kernel with dimensions $L \times L$ that weights the differences between pixels' intensity. The function $e(x, y): \mathbb{N}^{2} \rightarrow \mathbb{R}$ measures the distinctiveness between the pixel $p=(x, y)$ and its neighborhood of radius $L / 2$ :

$$
\begin{aligned}
& e(x, y)=\sum_{i=-L / 2}^{L / 2} \sum_{j=-L / 2}^{L / 2}[ {[\|I(x+i, y+j)-I(x, y)\|} \\
&\left.\times G\left(i+\frac{L}{2}, j+\frac{L}{2}\right)\right]
\end{aligned}
$$


where $I(x, y)$ is the image intensity at location $(x, y)$ and $\|$.$\| denotes the abso-$ lute value. Based on this function, a pixel $p=(x, y)$ is considered a "primitive" if it has minimal $e()$ value in a neighborhood of radius $L / 2$.

$$
\operatorname{pr}(x, y)=\left\{\begin{array}{l}
1(x, y)=\min _{\frac{L}{2}} e\left(x_{i}, y_{j}\right) \\
0 \text { otherwise }
\end{array}\right.
$$

As illustrated by figure 2, this process gives a set of primitives with variable dimension. These constitute the vertices of the graph that represents the structure of the iris image.

\subsection{Relationship Between Primitives}

In this section we describe how the relationships between primitives are established, i.e., how to find the graph's set of edges. The rationale of our method is to link neighbor vertices with high difference between their $e()$ values. These directions correspond to regions in the iris image with visible changes in the average image intensity. Let $P=\left\{p_{1}, \ldots, p_{k}\right\}, p_{i}=\left(x_{i}, y_{i}\right)$, be a set of primitives and $d()$ the Euclidean distance. We create an edge from $p_{i}$ to $p_{j}$ if

$$
e\left(p_{j}\right)<e\left(p_{i}\right) \wedge \frac{e\left(p_{i}\right)-e\left(p_{j}\right)}{d\left(p_{i}, p_{j}\right)}>\frac{h\left(p_{i}, \frac{L}{2}\right)+h\left(p_{j}, \frac{L}{2}\right)}{2}
$$

where $h(p, r)$ is the image entropy of the window centered at pixel $p$ and with radius $r$. As illustrated by figure 2, for each iris image we obtain a correspondent directed graph used to distinguish between identities. It can be observed the high similarity between the graphs resultant of images of the same iris (figures $2 a$ and $2 b$ ). Also, evident differences can be observed in the graph resultant of an image of a different iris (figure 2c), either the number of vertices and the edges directions.

\subsection{Structural Matching}

Having analyzed some of the most cited graph matching proposals, we observed that they commonly enhance the vertices and edges adjacency and do not take into account the relative position of each vertex in the graph, which is very relevant for our purposes. In this compass, Blondel et al. [1] proposed an iterative method that measures the similarity between graphs based on the observation that "two graph elements are similar (either vertices or edges) if their neighborhoods are similar".

Our measure of similarity between graphs combines the method of [1] and the minimal distance between the relative position of the vertices (primitives) in each graph. Let $G_{1}\left(P_{1}, E_{1}\right)$ and $G_{2}\left(P_{2}, E_{2}\right)$ represent two directed graphs, with $P_{i}$ vertices and $E_{i}$ edges. The function $g()$ gives the dissimilarity between graphs and is used to conclude about the identity of the subjects from where the graphs were constructed.

$$
g\left(G_{1}, G_{2}\right)=\alpha v\left(G_{1}, G_{2}\right)+(1-\alpha) e\left(G_{1}, G_{2}\right)
$$




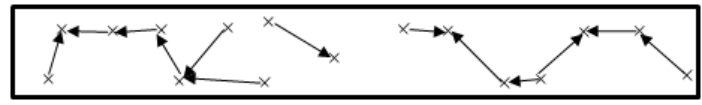

(a) Graph representing the structure the iris image "1" of subject "1".

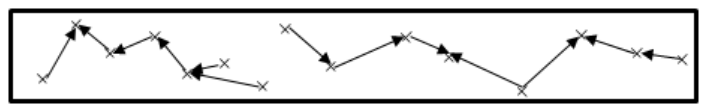

(b) Graph representing the structure of the iris image "2" of subject "1".

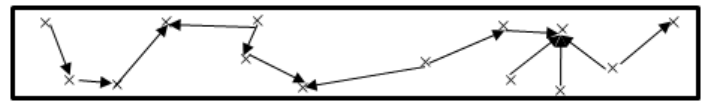

(c) Graph representing the structure of an iris image of subject " 2 ".

Fig. 2. Graphs that represent the structures of the iris images. The upper two figures illustrate the structures of different iris images of one subject and their similarity is evident. Oppositely, the bottom figure represents the structure of an iris image of a different subject and the dissimilarity is notorious.

where $0 \leq \alpha \leq 1$, can be used to weight the contribution of each term. Through trial-and-error, we empirically considered $\alpha=0.6$. The function $v()$ gives the minimal distance between the relative position of the primitives in the graph

$$
v\left(G_{1}, G_{2}\right)=\sum_{p_{i}} \min \frac{d\left(p_{i}, p_{j}\right)}{|P 1|}+\sum_{p_{j}} \min \frac{d\left(p_{j}, p_{i}\right)}{\left|P_{2}\right|}
$$

where $d()$ is the Euclidean distance, $|P|$ gives the cardinality of the $P$ set, $p_{i} \in P_{1}$ and $p_{j} \in P_{2}$.

As described in [1], the computation of the dissimilarity between graphs $G_{1}$ and $G_{2}$ produces a similarity matrix $M$ with dimension $\left|P_{1}\right| \times\left|P_{2}\right|$, where each cell $M(i, j)$ gives the dissimilarity between the $i^{t h}$ vertex of $G_{1}$ and the $j^{t h}$ vertex of $G_{2}$. The function $e()$ gives the minimal distance between elements of $M$ :

$$
e(G 1, G 2)=e(M)=\sum_{i} \min M(i, j)+\sum_{j} \min M(i, j)
$$

where $i=\left\{1, \ldots,\left|P_{1}\right|\right\}$ and $j=\left\{1, \ldots,\left|P_{2}\right|\right\}$. 


\section{Experiments and Discussion}

\subsection{Description of Experiments}

To compare the result obtained by the above described method, we implemented the statistical recognition algorithm proposed by Daugman [3]. This method is usually used as main comparison term for new iris recognition proposals and is the basis of all the commercially deployed iris recognition systems.

It starts by the the iris segmentation, through the integro-differential operator $\max _{r, x_{0}, y_{0}}\left|G_{\sigma}(r) * \frac{\delta}{\delta r} \oint_{r, x_{0}, y_{0}} \frac{I(x, y)}{2 \pi r} d s\right|$. This searches over the image domain $(x, y)$ for the maximum in the blurred partial derivative with respect to increasing radius $r$, of the normalized contour integral of $I(x, y)$ along a circular arc $d s$ of radius $r$ and center coordinates $\left(x_{0}, y_{0}\right)$. Further, to compensate the varying size of the pupil and capturing distances, images are normalized through the previously described Daugman Rubber Sheet. The coding of the iris data is accomplished through the use of bidimensional Gabor filters. These spatial filters have the form $G(x, y)=e^{-\pi \Phi} \cdot e^{-2 \pi i \Psi}$, with $\Phi=\left[\left(x-x_{0}\right)^{2} / \alpha^{2}+\left(y-y_{0}\right)^{2} / \beta^{2}\right]$ and $\Psi=\left[u_{0}\left(x-x_{0}\right)+v_{0}\left(y-y_{0}\right)\right] .\left(x_{0}, y_{0}\right)$ defines the position in the image, $(\alpha, \beta)$ is the filter width and length and $\left(u_{0}, v_{0}\right)$ specify the modulation, having spatial frequency $w_{0}=\sqrt{u_{0}^{2}+v_{0}^{2}}$ and direction $\theta_{0}=\arctan \left(v_{0} / u_{0}\right)$. To achieve invariance to illumination, the real parts of the 2-D Gabor filters are truncated to zero volume and the sign of the real and imaginary parts from quadrature image projections quantized into binary values. Finally, the Hamming distance is used in the computation of the dissimilarity between iris signatures.

Regarding the choused data sets, there are presently seven public and freely available iris image databases for biometric purposes: CASIA [6], Multimedia University (MMU), University of Bath, UPOL, Iris Challenge Evaluation [8], West Virginia University and UBIRIS. According to the characteristics of each one and to our purposes, we selected two data sets from the most widely used databases for biometric purposes: ICE and CASIA (third version). It should be stressed that the selected images, specially those from the $I C E$ database, contain significant noisy regions, ether due to iris obstructions (eyelids or eyelashes) or reflections (specular or lighting). Each data set contains 800 images from 80 subjects, enabling, respectively, 1800 and 78000 intra- and inter-class comparisons. Further, we divided each one into two halves. The first data sets - $C A S I A_{t r}$ and $I C E_{t r}$ - were used as training data and the later - $C A S I A_{t t}$ and $I C E_{t t}$ - to evaluate the recognition accuracy.

\subsection{Results}

Figure 3 contains a comparison between the results obtained by the statistical Daugman's method and our structural proposal, in the $C A S I A_{t t}$ (upper row) and $I C E_{t t}$ (bottom row) data sets. The histograms give the probability for the dissimilarity values between the intra- (bright series) and inter-class (dark series) comparisons. Also, the $\tau$ values correspond to the value of a Fisher-ratio test (FR) given by 


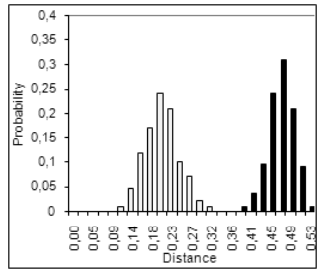

(a) Daugman's method, CAS I A tt data set, $\tau=191.39$.

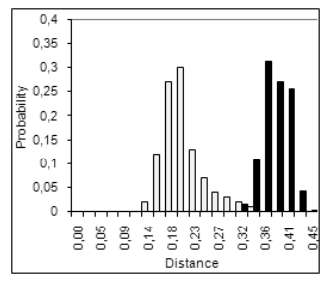

(d) Daugman's method, $I C E_{t t}$ data set, $\tau=97.11$.

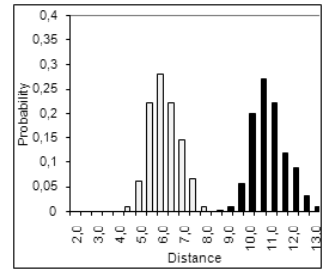

(b) Proposed method, CASI $A_{t t}$ data set, $\tau=161.80$.

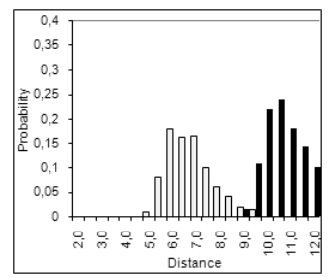

(e) Proposed method, $I C E_{t t}$ data set, $\tau=91.58$

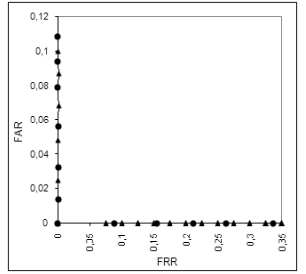

(c) ROCs obtained in the CASI Att data set.

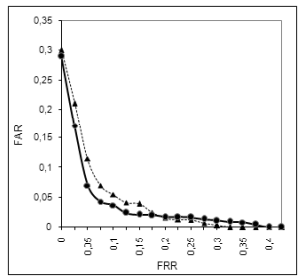

(f) ROCs obtained in the $I C E_{t t}$ data set.

Fig. 3. Comparison between the results (histograms and ROC's) obtained by the Daugman's algorithm (statistical PR) and our proposal (structural PR) in the $C A S I A_{t t}$ (upper row) and $I C E_{t t}$ (bottom row) data sets.

$\left(\mu_{E}-\mu_{I}\right)^{2} /\left(\frac{\sigma_{I^{2}}^{2}}{t_{I}}+\frac{\sigma_{E}{ }^{2}}{t_{E}}\right)$, where $\mu_{I}$ and $\mu_{E}$ respectively denote the average of the intra- and inter-class comparisons. $\sigma_{I}$ and $\sigma_{E}$ are the respective standard deviations and $t_{I}$ and $t_{E}$ the total of intra- and inter-class comparisons. Finally, the figures of the right column (figures $3 \mathrm{c}$ and $3 \mathrm{f}$ ) are the obtained receiver operating curves (ROCs) by the Daugman's method (continuous series) and our proposal (dashed series).

Both recognition methods achieved complete separability between the intra- and inter-class comparisons in the $C A S I A_{t t}$ data set and presented error rates equal to 0 . Thus, the obtained ROCs fall into the horizontal and vertical axes. Regarding the noisier data set (ICE), the obtained error rates were similar, although the (Fr) values indicate a slightly higher separability between classes by the Daugman's method. However, our proposal achieved a lower value for the false rejections (FRR), when the false acceptances (FAR) were minimized (FRR, FAR $=0$ ), which is very relevant, as this is a measure commonly used for the comfort that recognition systems give to the users. These experiments led us to conclude that, with further optimization, our proposal can constitute an alternative to statistical PR approaches. 


\section{Conclusions and Further Work}

In this paper we proposed an iris recognition algorithm which, singularly, follows the structural PR paradigm. Our experiments were performed on two widely used iris image databases (CASIA.v3 and ICE) and show that the proposed iris structure contains enough information to accurately distinguish between individuals. The structural pattern recognition approach reveals itself advantageous essentially to improve the human-perception and description of the PR system's functioning, which can be relevant, for instance, for security / forensic purposes.

Our current work is focused in the evaluation of the robustness that structuralbased iris recognition algorithms have to noisy images. We hope to find isomorphisms between sub-graphs, in order to perform biometric recognition based exclusively in the information extracted from small portions of the iris texture, which will substantially increase the operability of iris recognition within noisy image capturing environments.

\section{References}

1. V. Blondel, A.Gajardo, M. Heymans, P. Senellart, and P. Dooren (2004) A measure of similarity between graph vertices: applications to synonym extraction and web searching, SIAM Review 46, 4:647-666

2. W. W. Boles and B. Boashash (1998) A human identification technique using images of the iris and wavelet transform, IEEE Transactions on Signal Processing 46, 4: 1185-1188

3. J. G. Daugman (1993) High confidence visual recognition of persons by a test of statistical independence, IEEE Transactions on Pattern Analysis and Machine Intelligence, 25, 11: $1148-1161$

4. E. R. Dougherty (1992) An Introduction to Morphological Image Processing, SPIE Optical Engineering Press

5. Y. Huang, S. Luo, and E. Chen (2002) An efficient iris recognition system, In Proceedings of the First International Conference on Machine Learning and Cybernetics:450-454.

6. Institute of Automation, Chinese Academy of Sciences (2004) CASIA iris image database, http://www.sinobiometrics.com

7. L. Ma, T. Tan, D. Zhang, and Y. Wang (2004) Local intensity variation analysis for iris recognition, Pattern recognition, 37, 6: 1287-1298

8. National Institute of Standards and Technology (2006) Iris challenge evaluation, http://iris.nist.gov/ICE/

9. R. P. Wildes (1997) Iris recognition: an emerging biometric technology, Proceedings of the IEEE, 85, 9: 1348-1363 